\title{
Scrub Typhus in Western Nepal: A Series of Case Reports
}

\author{
Pudasaini $\mathbf{P}^{1}$, Ranjit $\mathrm{A}^{2}$, Neupane $\mathbf{S}^{3}$
}

${ }^{1}$ Resident, ${ }^{2}$ Lecturer, ${ }^{3}$ Professor, Department of Dermatology, Gandaki Medical College, Pokhara, Nepal.

\begin{abstract}
Scrub typhus, a mite borne infectious disease which is transmitted to humans by bite of trombiculid mite. An eschar, which is a characteristic necrotic skin lesion, is formed at the site of bite. Systemic spread via the hematogenous and lymphatic routes occur and the infected people develop fever, cutaneous rash, myalgia, and lymphadenopathy. Diagnosis of scrub typhus is often difficult because of vast variability and non-specific presentation of the disease. Scrub typhus has been poorly reported from Kaski district. Here we report series of five cases of scrub typhus from Kaski distrist of Western Nepal.
\end{abstract}

Key words: Nepal; Orientia tsutsugamushi; Scrub Typhus; Trombiculidae

\section{Introduction}

Crub typhus, also known as tsutsugamushi disease, is caused by Orientia tsutsugamushi, which is an arthropod-borne gram-negative obligately intracellular bacillus. ${ }^{1-3}$ Bites of infected larval stage of the trombiculid mites transmit scrub typhus to humans. ${ }^{4}$ When the pathogen enters the human body, proliferation of the pathogen occurs at the site of bite, and an eschar, which is a characteristic necrotic skin lesion, is formed at the site of bite. Systemic spread via the hematogenous and lymphatic routes occur and the infected people develop fever, cutaneous rash, myalgia, and lymphadenopathy. Scrub typhus is difficult for the clinicians to diagnose because of the vast variability and non-specific presentation of the disease. ${ }^{5}$ Scrub typhus has been poorly reported from Kaski district with only five cases among a total of 76 cases from Gandaki province according to the data from epidemiology and disease control division of Nepal, 2019. ${ }^{6}$ Here we report five cases of Scrub typhus, all of the cases with presence of pathognomic eschar, four of which had negative scrub typhus serology, but all the cases responded well with the treatment regimen of scrub typhus.

Financial disclosure: None.

Conflict of interest to disclosure: None declared.

\section{Address of Correspondence}

Dr. Prajwal Pudasaini

Resident

Department of Dermatology

Gandaki Medical College, Pokhara, Nepal.

E-mail: prajwalpudasaini@gmail.com

\section{Case Report}

\section{Case 1}

A 34-year old female from Syangja, farmer by occupation, presented with complaints of solitary lesion over skin of upper anterior abdomen for 10 days and fever for three days. Initially, three days after insect bite, a small papule with erythema, edema in surrounding area with eschar, appeared which was associated with pain and fever. Fever occurred four days after insect bite and was associated with chills and rigor. On examination, the patient was febrile with temperature of $102^{\circ} \mathrm{F}$ and pulse rate of 50/ min (relative bradycardia). There was no history of joint pain, vomiting, altered sensorium, yellowish discoloration of body, shortness of breath, dyspnoea, syncope or changes in bowel and bladder habit.

On cutaneous examination: A $2 \times 1 \mathrm{~cm}$ plaque with well-defined margin, smooth surface, central eschar, and crust with erythematous surrounding was present in abdomen, $5 \mathrm{~cm}$ from xiphisternum. Regional lymphadenopathy was absent (figure 1).

Submitted: $30^{\text {th }}$ October 2019

Accepted: $1^{\text {st }}$ December 2019

Published: $7^{\text {th }}$ October 2020

How to cite this article

Pudasaini P, Ranjit A Neupane S. Scrub typhus in Western Nepal: A series of case reports. Nepal Journal of Dermatology, Venereology and Leprology 2020;18(1):67-72. https://doi. org/10.3126/njdvl.v18i1.27290.

Licensed under CC BY 4.0 International License which permits use, distribution and reproduction in any medium, provided the original work is properly cited. 
Investigations: WBC count was $9800 / \mathrm{mm}^{3}$, Platelet was $15400 / \mathrm{mm}^{3}$, SGOT was $298 \mathrm{IU} / \mathrm{ml}$, SGPT was 312 $\mathrm{IU} / \mathrm{ml}$, ALP was $613 \mathrm{IU} / \mathrm{ml}$, Ultrasound of Abdomen/ Pelvis showed splenomegaly. Serum tsutsugamushi antibody assay was negative, however, IgM antibody, weilfelix test, Polymerase Chain Reaction (PCR) were not done. Patient was treated with Doxycycline $100 \mathrm{mg}$ po bd for 14 days. Fever subsided within 24 hours of oral doxycycline administration.

\section{Case 2}

A 32-year old male presented with a painful red lesion over left arm for three days. Initially, a small papule was present that enlarged with central blister, release of watery substance with formation of brownish crust with pain and erythema. He gave history of working in farm. On cutaneous examination, a $2 \times 3 \mathrm{~cm}$ plaque with a central vesicle, brownish black crust, and surrounding erythema over the flexor aspect of left upper arm, $2 \mathrm{~cm}$ from the elbow joint was present. Swelling and tenderness was also present. (Figure 2). Investigations :WBC, $\mathrm{Hb}$, Platelet counts were within normal limit. SGOT was $64 \mathrm{lU} / \mathrm{ml}$,SGPT was $52 \mathrm{lU} / \mathrm{ml}$, ALP was within normal range,USG Abdomen/Pelvis showed splenomegaly. Serum tsutsugamushi antibody assay was negative, however IgM antibody, weilfelix test, Polymerase Chain Reaction (PCR) were not done.

\section{Case 3}

A 38-year old female presented with a painful red lesion over left leg for 3 days. Initially, the lesion was small that later increased in size and spread to form cord like red painful lesion that extended to upper medial thigh. Pain was more on walking and movement of legs. Generalized body ache was present. Patient occasionally worked in a farm. On examination, a welldefined erythematous plaque of $2 \times 2 \mathrm{~cm}$ in size with a central brownish black crust, central erosion over left leg about $10 \mathrm{~cm}$ from left knee joint was present. Linear erythematous cord like plaque of $20 \times 2 \mathrm{~cm}$ with extension to upper medial thigh was present. Inguinal and popliteal lymph nodes were not enlarged and were nontender (figure 3). Investigations: Blood counts were normal. Liver enzymes were normal except a slightly increased ALP. Ultrasonography of abdomen and pelvis was normal. Serum tsutsugamushi antibody assay was negative, however IgM antibody, weilfelix test, Polymerase Chain Reaction (PCR) were not done. C. Doxycycline $100 \mathrm{mg}$ po bd was given and patient became afebrile within 24 hours of treatment.

\section{Case 4}

A 43-year old male was admitted with complaints of painful red lesion over left leg for 20 days and new painful red lesion over left leg for 3 days. Initially, a small red lesion was present that ruptured with release of watery fluid and formation of brownish black crust. Lesion increased in size and subsequently healed after application of topical antibiotic. New lesion was also small in size that enlarged. It was associated with pus point with formation of brownish crust in the centre, with associated swelling and redness around the lesion. Patient was a lecturer in an agriculture faculty with recent travel to Birtamod, Ilam and Chitwan.

He did not give any history of injury or trauma at the site of lesion.

On examination, the patient's general condition was fair and vitals were stable. Cutaneous examination revealed multiple plaques over left lower leg with largest one measuring $2 \times 2 \mathrm{~cm}$ plaque with central brownish black crust over left lower leg in the lateral aspect. Plaque with erythematous border and central erosion was also present over lateral aspect of left leg in the lower part. A crusted plaque of $2 \times 1 \mathrm{~cm}$ in size, 10 $\mathrm{cm}$ from ankle joint was also present in the left lower leg. Local temperature was raised and tenderness was present. Popliteal and inguinal lymph node were not enlarged and were non tender (figure 4).

Investigations showed normal range of white blood cells count, neutrophil, lymphocytes, eosinophil and platelet counts. SGOT and SGPT were also within normal range but ALP was slightly increased. $\mathrm{Na} / \mathrm{K}$, urea, creatinine was within normal range. Ultrasonography of abdomen/pelvis revealed fatty liver. Serum tsutsugamushi antibody assay was negative, however, IgM antibody, weilfelix test, Polymerase Chain Reaction (PCR) were not done.

Treatment with capsule Doxycycline $100 \mathrm{mg}$ po bd was given for 2 weeks along with Mupirocin ointment for two weeks. The patient responded well and became afebrile within 24 hours of treatment and there was post inflammatory hypopigmentation with complete resolution after one month of therapy.

\section{Case 5}

A 44 years old male from Damside, Pokhara, was admitted with complaints of fever for one week and painless lesion and swelling over left inguinal region for eight days. He developed fever with maximum temperature of $104^{\circ} \mathrm{F}$, associated with chills and rigor, four days after he had been to Fewa lake for fishing. Fever was associated with malaise, headache and anorexia. Initially, a small lesion was present which was elevated from the surface of skin. Lesion increased in size and formed blackish crust in the centre, later on there was redness in surrounding area with 
depressed centre. Swelling and redness appeared five days after fishing in fewa lake. It was painless and was associated with swelling over the inguinal region with no discharge or oozing. He did not give any history of lesion in penile region and scrotum. He did not give any history of unprotected sexual intercourse. He had frequent field visits and often went for fishing around fewa lake. There was no history of recent travel to endemic areas, injury or trauma at the site of lesion, insect bite, venous prominences, heaviness in legs, headache, vomiting, abdominal pain or diarrhea. Patient had normal bowel and bladder habit.

On examination, the patient's general condition was fair, vitals were stable and left inguinal lymph node was enlarged which was non tender.

Cutaneous examination revealed a plaque of approximately $2 \times 1 \mathrm{~cm}$ with a well-defined margin, smooth surface and central ulceration. Indurated ulcer with ulcer base containing serous discharge was

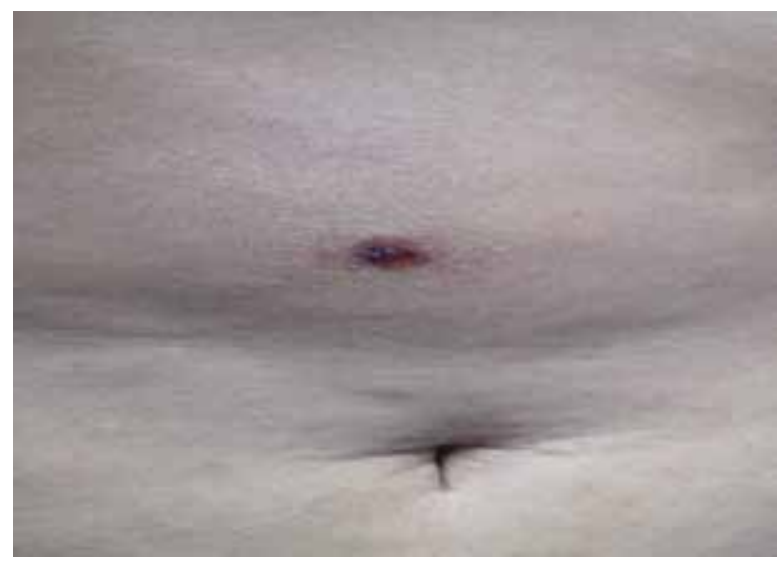

Figure 1: A 2x1 cm plaque with a well-defined margin, smooth surface, central eschar, and crust with erythematous surrounding over abdomen. present over the skin of upper medial aspect of left thigh approximately $5 \mathrm{~cm}$ below and medial to anterior superioriliac spine. Surrounding skin was erythematous with absence of swelling and tenderness. Left inguinal lymph node was enlarged but was non tender (figure 5).

Investigations: Blood counts, random blood sugar, urea, creatinine, sodium, potassium, liver enzymes were within normal range. Routine urine examination revealed normal findings. Ultrasonography of abdomen/pelvis revealed fatty liver, Chest Xray was normal. Serum tsutsugamushi antibody assay was positive, however IgM antibody, weilfelix test, Polymerase Chain Reaction (PCR) were not done, widal test was negative, Brucella antibody, Leptospira antibody, dengue antibody and malaria parasite antigen was negative. Blood and urine culture showed no growth. Treatment with capsule Doxycycline 100 $\mathrm{mg}$ po twice a day for 14 days was given and the patient improved.

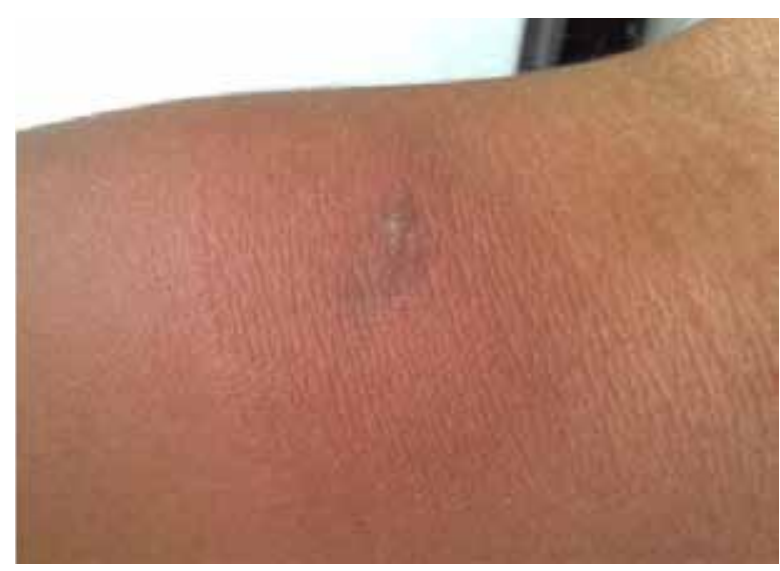

Figure 2: A $2 \times 3 \mathrm{~cm}$ plaque with a central vesicle, brownish black crust, surrounding erythema over the flexor aspect of left upper arm.

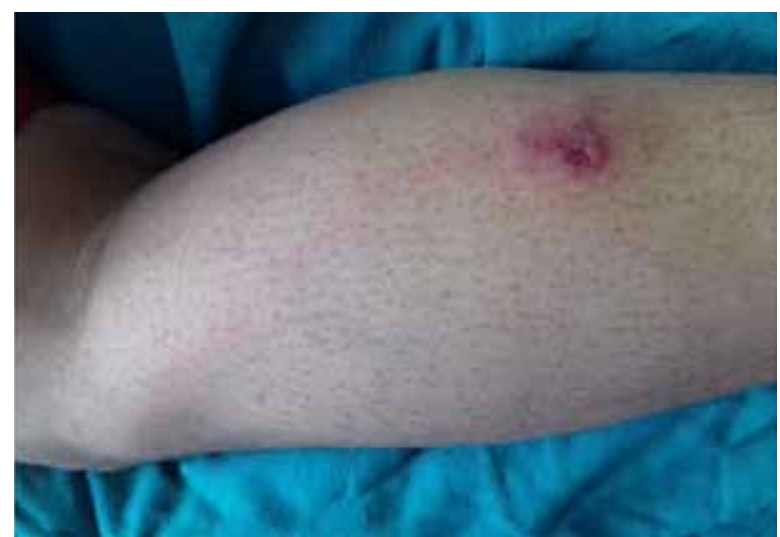

Figure 3: A well-defined erythematous plaque of $2 \times 2$ $\mathrm{cm}$ in size with central brownish black crust, central erosion over left leg with an erythematous cord like linear track extending up to upper medial thigh. 


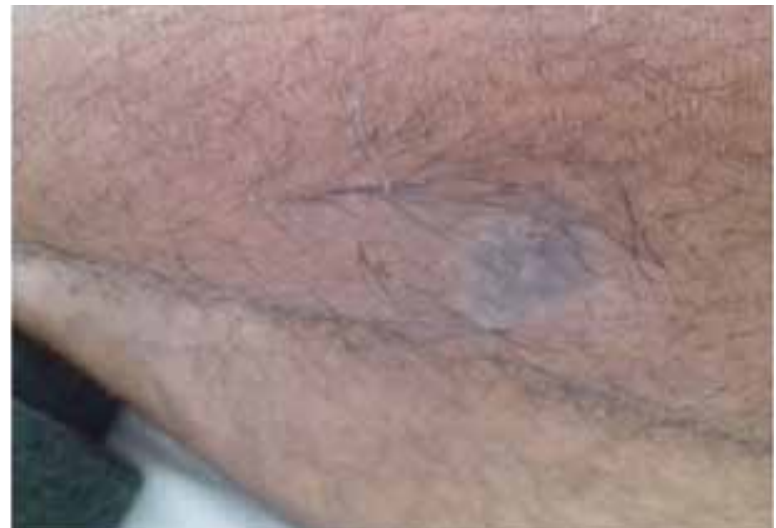

Figure 4: (a) before treatment, a $2 \times 2 \mathrm{~cm}$ plaque with central brownish black crust over left lower leg in the lateral aspect.

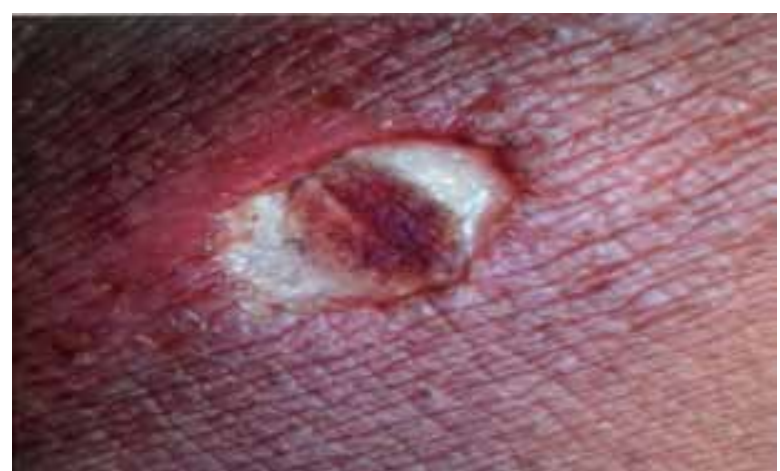

Figure 5: (a) before treatment, a $2 \times 1 \mathrm{~cm}$ plaque with well defined margin, smooth surface and central ulceration over upper medial aspect of left thigh.

\section{Discussion}

Scrub typhus, also known as tsutsugamushi disease, is caused by Orientia tsutsugamushi, which is an arthropod-borne gram-negative, obligately intracellular bacillus. ${ }^{1,2,3}$ Bites of infected larval stage of the trombiculid mite transmits scrub typhus to humans. ${ }^{4}$ The incubation period of the disease is about 5 to 20 days. Systemic spread via the hematogenous and lymphogenous routes occur and the infected people develop fever, cutaneous rash, myalgia, and lymphadenopathy. ${ }^{5}$

Acute febrile illness with non-specific signs and symptoms such as fever, myalgia, headache, abdominal pain, vomiting, loose motions, conjunctival congestion, generalized weakness, and jaundice are common manifestations of scrub typhus. ${ }^{7,8}$ Other diseases such as enteric fever, leptospirosis, dengue and murine typhus can also have similar presentation. ${ }^{9}$

Scrub typhus is endemic in the geographical tsutsugamushi triangle that extends from far-eastern Russia and northern Japan in the north, to the south

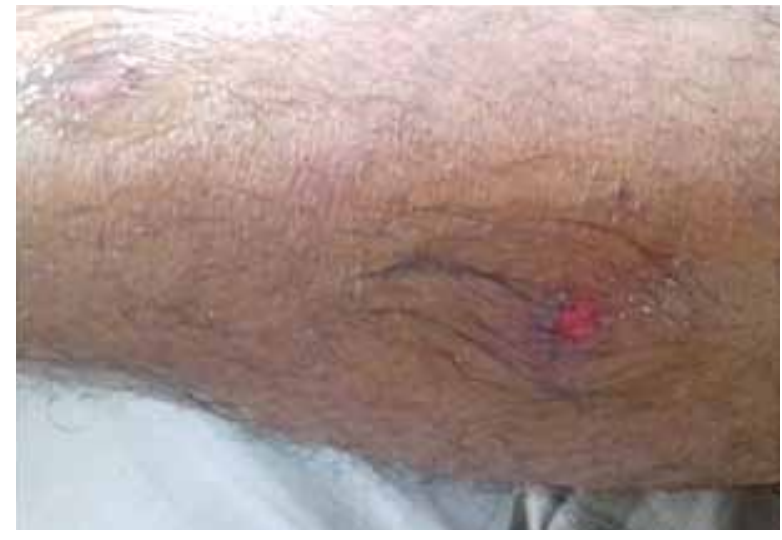

Figure 4: (b) after treatment.

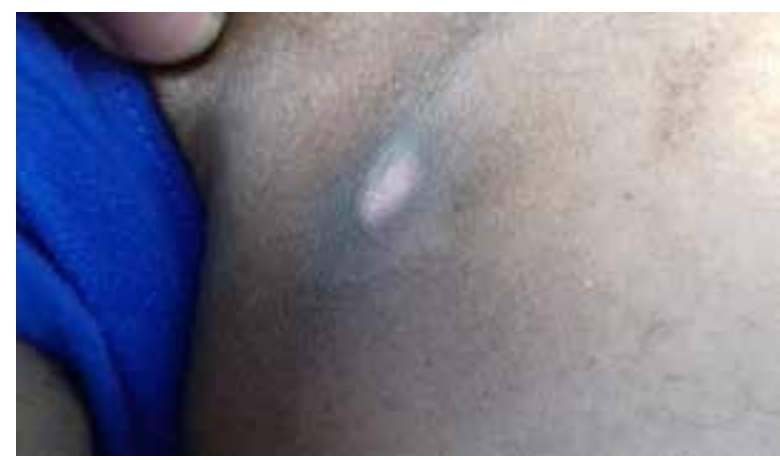

Figure 5: (b) after treatment.

(territories around the Solomon Sea into northern Australia) and to Pakistan and Afghanistan in the west. Nepal is also within the tsutsugamushi triangle. In Southeast Asia, scrub typhus is a leading cause of treatable non-malarial febrile illness. ${ }^{10}$ Diagnostic facilities is not available and the precise incidence of the scrub typhus is also unknown. An increase in $\geq$ four-fold in scrub typhus antibody titer is used for diagnosis of scrub typhus. ${ }^{11}$ The oldest Weil-Felix OX-K agglutination test lacks sensitivity and specificity. ${ }^{12}$ Identification of Rickettsia tsutsugamushi antibody by enzyme-linked immunosorbent also has high diagnostic sensitivity (95\%) and specificity (100\%). ${ }^{13}$

Although we did not get positive serology in all of the cases, all the cases had a typical eschar and all of them responded promptly to treatment with Doxycycline which gave us a diagnostic clue.

Scrub typhus is one of the re-emerging diseases in Nepal. There was a singular spike in cases of scrub typhus after the earthquake in $2015 .{ }^{14}$ Although fever is one of the commonest presenting complaint, many cases of pyrexia of unknown origin, due to lack 
of appropriate laboratory facilities, remains poorly differentiated. ${ }^{15}$ Our second case even gave a picture of enteric fever. The clinical suspicion of scrub typhus is still low, and often false diagnosis is made, as fever may go unnoticed and eschar and typical rash are not always present. ${ }^{16}$ One of our cases even gave a picture of enteric fever. But it was not responding to antibiotics and the presence of a typical eschar gave us a clue of scrub typhus. We treated the case with Doxycycline and the response was prompt, the patient became afebrile after 24 hours. Scrub typhus has been poorly reported from Gandaki province with five cases from kaski, 14 cases from Syangja, four cases from Parbat, 16 cases from Gorkha, five cases from Tanahun, two cases from Lamjung, 14 cases from Baglung and 16 cases from Nawalparasi among a total of 76 cases from Gandaki province, according to the data from Epidemiology and disease control division of Nepal, 2019. ${ }^{6}$

\section{References}

1. Valbuena $G$, Walker D. Approaches to vaccines against Orientia tsutsugamushi. Frontiers in Cellular and Infection Microbiology 2013;2. https://doi.org/10.3389/fcimb.2012.00170

2. Kim IS, Walker DH. Scrub Typhus. In: Guerrant RL, Walker DH, Weller PF, editors. Tropical infectious diseases: principles, pathogens and practice. 3rd ed: Elsevier Health Sciences;2011.p. 332-6. https://doi.org/10.1016/B978-0-7020-39355.00051-3

3. Paris DH, Shelite TR, Day NP, Walker DH. Unresolved problems related to scrub typhus: A seriously neglected life-threatening disease. Am J Trop Med Hyg 2013;89: 301-7. https://doi. org/10.4269/ajtmh.13-0064

4. World Health Organization. Frequently Asked Questions Scrub Typhus. 2013[Internet]. [Accessed on 9th October 2019]. Available from: http://www.searo.who.int/entity/emerging_ diseases/CDS_faq_Scrub_Ty

5. Li T, Yang Z, Dong Z, Wang M. Meteorological factors and risk of scrub typhus in Guangzhou, southern China, 2006- 2012. BMC Infect Dis 2014; 14: 139 . https://doi.org/10.1186/14712334-14-139

6. Epidemiology and Disease Control Division (EDCD). Scrub Typhus update, 2019 [Internet]. [Accessed 7th December 2019]. Available from: http://www.edcd.gov.np/news/download/ scrub-typhus

7. Singh SP, Singh $R$ and Ahmad N. A study of complications of scrub typhus in a tertiary
As scrub typhus is re-emerging and clinicians are also less aware about scrub typhus prevalence, awareness about disease, its consequences and preventive measures need to be started.

\section{Conclusion}

As there can be variable modes of presentation of scrub typhus, with no widely available diagnostic modality for the appropriate identification and timely intervention of the disease, sensitive and specific diagnostic test needs to be employed at all level of health centers and hospitals to diagnose any patients with acute febrile illness for scrub typhus and provide timely intervention and prevent the complications.

health care institute of Uttarakhand, India. Int J Res Med Sci 2014;2(1):246-49. https://doi. org/10.5455/2320-6012.ijrms20140247

8. Bhat NK, Dhar M, Mittal G, Shirazi N, Rawat A, Kalra BP, et al. Scrub typhus in children at a tertiary hospital in North India: clinical profile and complications. Iran J Pediatr 2014;24(4): 387-92.

9. Basnyat B. Typhoid versus typhus fever in post-earthquake Nepal. Lancet Glob Health 2016Aug;4(8):e516-17. https://doi.org/10.1016/ S2214-109X(16)30094-8

10. Acestor N, Cooksey R, Newton PN, Menard D, Guerin PJ, Nakagawa J, et al. Mapping the aetiology of non-malarial febrile illness in Southeast Asia through a systematic reviewterra incognita impairing treatment policies. PLoS One 2012;7(9):6. https://doi.org/10.1371/ journal.pone.0044269

11. Blacksell SD, Bryant NJ, Paris DH, Doust JA, Sakoda Y, Day NPJ. Scrub typhus serologic testing with the indirect immunofluorescence method as a diagnostic gold standard: a lack of consensus leads to a lot of confusion. Clin Infect Dis 2007;44:391-401. https://doi. org/10.1086/510585

12. Kelly DJ, Wong PW, Gan E, Lewis GE. Comparative evaluation of the indirect immunoperoxidase test for the serodiagnosis of rickettsial disease. Am J Trop Med Hyg 1988;38:400-6. https://doi. org/10.4269/ajtmh.1988.38.400

13. Kim IS, Seong SY, Woo SG, Choi MS, Chang WH. High-level expression of a 56-kilodalton protein gene (bor56) of Rickettsia tsutsugamushi 
Boryong and its application to enzyme-linked immunosorbent assays. J Clin Microbiol 1993; 31:598. https://doi.org/10.1128/JCM.31.3.598605.1993

14. Basnyat B. Aftershocks of scrub typhus in Nepal-Author's reply. Lancet Glob Health 2016; 4(10): e688. https://doi.org/10.1016/S2214109X(16)30208-X

15. Murdoch DR, Woods CW, Zimmerman MD, Dull $\mathrm{PM}$, Belbase RH, Keenan AJ, et al. The etiology of febrile illness in adults presenting to Patan Hospital in Kathmandu Nepal. Am J Trop Med Hyg 2004;70:670-5. https://doi.org/10.4269/ ajtmh.2004.70.670

16. Thompson CN, Blacksell SD, Paris DH, Arjyal A, Karkey A, Dongol S, et al. Undifferentiated febrile illness in Kathmandu Nepal. Am J Trop Med Hyg 2015;92:875-8. https://doi.org/10.4269/ ajtmh.14-0709 